\title{
Transporte aéreo de pacientes: análise do conhecimento científico
}

\author{
Air transport of patients: analysis of scientific knowledge \\ Transporte aéreo de pacientes: análisis del conocimiento científico
}

Isis Pienta Batista Dias Passos', Vanessa Pellegrino Toledo", Erika Christiane Marocco Duran"

' Força Aérea Brasileira, Academia da Força Aérea, Subdivisão de Saúde. Pirassununga -SP, Brasil.

" Universidade Estadual de Campinas, Faculdade de Ciências Médicas, Departamento de Enfermagem. Campinas-SP, Brasil.

Submissão: 10-09-2010 Aprovação: 07-12-2011

\section{RESUMO}

Pesquisa bibliográfica, cujo objetivo foi caracterizar a produção científica acerca do transporte aéreo de pacientes, em bases de dados indexadas. Encontrou-se 83 referências, 76 na Medline, entre 1997 a 2009, e quatro na Lilacs, de 1999 a 2007. Verificouse maior publicação em 2001, sendo a pesquisa qualitativa mais prevalente. A tendência temática mais evidenciada na Lilacs foi educação e pesquisa. Na Medline foi cuidado de enfermagem no transporte aéreo. Destaca-se escassa regulamentação no que se refere as atribuições do enfermeiro de bordo e a importância da inclusão do conteúdo de enfermagem aeroespacial nos cursos de graduação e pós-graduação. Existem ainda muitas lacunas na produção de conhecimento sobre o tema. Pesquisas que analisem esta interface, qualificam o cuidado de enfermagem aeroespacial.

Descritores: Enfermagem militar; Enfermagem em emergência; Resgate aéreo; Transporte de pacientes; Medicina aeroespacial.

\section{ABSTRACT}

This bibliographic research aimed to characterize scientific production on air patient transport in indexed databases. Eightythree references were found, 76 in Medline, between 1997 and 2009, and four in Lilacs, between 1999 and 2007. More publications were found in 2001 and qualitative studies were the most prevalent. The most evident thematic tendency in Lilacs was education and research, against air transport nursing in Medline. The lack of regulation on nurse's tasks on board is highlighted, as well as the importance of including contents on aerospace nursing in undergraduate and graduate courses. Many gaps remain in knowledge production about the theme. Research analyzing this interface qualifies aerospace nursing care. Key words: Military nursing; Emergency nursing; Air ambulances; Transportation of patients; Aerospace medicine.

\section{RESUMEN}

Investigación bibliográfica con objeto de caracterizar la producción científica acerca del transporte aéreo de pacientes en bases de datos indexadas. Fueron encontradas 83 referencias, 76 en Medline, entre 1997 y 2009 , y cuatro en Lilacs, de 1999 a 2007. Fue verificada mayor publicación en 2001, con mayor prevalencia de la investigación cualitativa. La tendencia temática más evidenciada en Lilacs fue educación e investigación. En Medline fue cuidado de enfermería en el transporte aéreo. Se destaca escasa reglamentación respecto a las atribuciones del enfermero de bordo y la importancia de la inclusión del contenido de enfermería aeroespacial en los cursos de pregrado y postgrado. Todavía existen muchas lagunas en la producción de conocimiento sobre el tema. Investigaciones que analicen esta interfaz cualifican el cuidado de enfermería aeroespacial. Palabras clave: Enfermería militar; Enfermería de urgencia; Ambulancias aéreas; Transporte de pacientes; Medicina aeroespacial. 


\section{INTRODUÇÃO}

Estudos acerca do transporte aéreo de pacientes demonstram uma preocupação sobre a segurança, focando os riscos para os pacientes. Entretanto, poucos enfocam a assistência de enfermagem realizada durante o transporte do paciente, considerado um intervalo de tempo crucial para minimizar as complicações e representa uma área de investigação importante para a segurança do paciente ${ }^{(1)}$.

O uso de helicópteros médicos, como uma estratégia para vencer o tempo e as barreiras geográficas de acesso ao atendimento, continua a crescer. O número de aeronaves médicas duplicou na última década nos Estados Unidos, Canadá e Europa. Embora não haja continuidade do debate sobre a adequação de helicópteros médicos, destaca-se a importância dos cuidados de enfermagem na hora de ouro, pois estudos comprovam que as longas distâncias de vôo foram associadas ao aumento da incidência de eventos $\operatorname{adversos}^{(1)}$.

Sendo assim, fazem-se necessários estudos acerca do transporte aéreo de pacientes, haja vista as complicações decorrentes das longas distâncias de vôo e a efetividade da assistência de enfermagem que interferem diretamente no sucesso do atendimento.

Na Força Aérea Brasileira (FAB) há constantemente missões de Evacuação Aeromédica (EVAM) ou Missões de Misericórdia (MMI), em que é feito o transporte aéreo de pacientes para locais com melhores recursos. Os médicos em sua formação militar têm em seu currículo a disciplina Medicina Aeroespacial. Já a enfermagem, infelizmente adquire o conhecimento durante o próprio transporte aéreo, ou seja, na prática do trabalho.

O transporte aéreo vem obtendo destaque, desde seu início em 1870, na Guerra Franco Prussiana. Depois, foi utilizado na Segunda Guerra Mundial e nas Guerras da Coréia e Vietnã ${ }^{(2)}$. Neste tipo de transporte se faz necessário a presença do enfermeiro e, de acordo com a Emergency Nurses Association (ENA), é importante que este profissional seja treinado e tenha experiência na assistência a pacientes graves $^{(3)}$

No Brasil, a remoção aeromédica teve início na FAB, com o Serviço de Busca e Salvamento (SAR), em 1950. Depois, muitos outros locais implantaram esse tipo de remoção, por exemplo, o Corpo de Bombeiros Militares do Rio de Janeiro e o Projeto Resgate do Estado de São Paulo(3). O antigo DAC (Departamento de Aviação Civil), hoje ANAC (Agência Nacional de Aviação Civil), é o órgão responsável por homologar as aeronaves para esse tipo de remoção, bem como supervisionar e estabelecer um currículo mínimo para a formação e treinamento da tripulação aeromédica(3).

Há uma série de requisitos que o enfermeiro de bordo deve ter, dentre eles: boa condição física, controle emocional, criatividade, habilidade em improvisar, porém há poucas disciplinas e/ou cursos específicos no Brasil(3).

Este estudo tem como objetivo caracterizar a produção científica acerca do transporte aéreo de pacientes, em bases de dados indexadas.

\section{MÉTODOS}

Trata-se de uma pesquisa bibliográfica sobre a produção científica do transporte aéreo de pacientes. A pesquisa bibliográfica pode ser realizada como parte da pesquisa descritiva ou experimental, ou independentemente. Busca conhecer e avaliar um problema a partir de referências teóricas publicadas em documento ${ }^{(4)}$. Tem como objetivo encontrar respostas aos problemas, e o recurso utilizado é a consulta aos documentos bibliográficos. Tem por finalidade colocar o pesquisador em contato com o que já se produziu a respeito do tema pesquisado $^{(5)}$.

O estudo foi realizado nas bases de dados LILACS (Literatura Latino Americana e do Caribe em Ciências da Saúde) no período compreendido entre 1988 a 2007, e MEDLINE (Bibliografia Médica) de 1997 a 2009.

O critério de escolha dos artigos e resumos foi possuir pelo menos dois dos descritores: enfermagem militar, enfermagem, papel do profissional de enfermagem, enfermagem em emergência, educação em enfermagem, transporte aéreo, resgate aéreo, transporte de pacientes, medicina aeroespacial.

Após a seleção dos artigos, foi realizada a leitura analítica do material, classificando-os de acordo com o ano de publicação, o periódico em que está divulgado, o delineamento metodológico e a tendência temática abordada no estudo.

A análise do delineamento metodológico das pesquisas foi realizada com base no referencial descrito por Polit, Beck e Hungler, através das categorias de estudos quantitativos (experimental, quase-experimental e não-experimental) e qualitativos (etnografia, fenomenologia e teoria fundamentada) ${ }^{(6)}$.

Utilizou-se a planilha do programa computacional Excel para a inserção dos dados. A análise foi descritiva e quanti-qualitativa.

\section{RESULTADOS E DISCUSSÃO}

Nas bases de dados pesquisadas foram encontradas 83 referências, sendo 76 na Medline, entre 1997 a 2009, e quatro na Lilacs, de 1999 a 2007. Pela falta de acesso aos resumos, foram analisadas 33 publicações da Medline.

Na Tabela 1 observa-se que no ano de 2001 a distribuição dos resumos na base de dados Medline foi de 15,16\% $(n=5)$, e na Lilacs, no período estudado, foi de quatro, distribuídos nos anos de 1999, 2000, 2003 e 2007.

A partir de 1997 destaca-se a produção científica sobre a enfermagem aeromédica, que corroborou com a necessidade do enfermeiro de bordo, um profissional especializado em transporte de pacientes aerorremovidos. No Brasil, ainda é uma atividade recente, não existindo disciplina ou curso específico. O treinamento desse enfermeiro é oferecido pela instituição que realiza a remoção aeromédica e supervisionado pela Agência Nacional de Aviação Civil (ANAC) ${ }^{(3)}$.

A ANAC estabelece um currículo mínimo para o treinamento da tripulação aeromédica, composta por médico e enfermeiro de bordo, segundo a legislação vigente de 1996. São obrigatórios conhecimentos acerca de: aspectos fisiológicos em vôo; embarque e desembarque de pacientes; segurança 
Tabela 1 - Distribuição dos resumos nas bases de dados Medline e Lilacs segundo o ano de publicação, 1997 a 2008.

\begin{tabular}{llcl} 
& Medline & Lilacs & Total \\
Ano & $\mathbf{n}$ & $\mathbf{n}$ & $\mathbf{n}$ \\
1997 & 1 & - & 1 \\
1998 & 3 & - & 3 \\
1999 & 4 & 1 & 5 \\
2000 & 3 & 1 & 4 \\
2001 & 5 & - & 5 \\
2002 & 3 & - & 3 \\
2003 & 2 & 1 & 3 \\
2004 & 3 & - & 3 \\
2005 & 3 & - & 3 \\
2006 & 3 & - & 3 \\
2007 & 2 & 1 & 3 \\
2008 & 1 & - & 37 \\
Total & 33 & 4 & \\
\hline
\end{tabular}

no interior e em torno da aeronave; instrução aos passageiros; procedimentos apropriados de emergência em vôo; de pousos de emergência e de evacuação de emergência. Estabelece ainda, um programa mínimo de formação, com curso de Medicina Aeroespacial Aplicada ao Transporte Aeromédico, com a finalidade de preparar melhor a tripulação aeromédica ${ }^{(3)}$.

A Emergency Nurses Association e a National Flight Nurses Association, dos EUA, recomendam que sempre haja um enfermeiro treinado na equipe de remoção aeromédica. No Brasil, a Lei do Exercício Profissional $\mathrm{n}^{\circ} 7498 / 86$, estabelece que é privativo do enfermeiro a organização e direção de áreas críticas e a assistência direta à pacientes críticos. Os requisitos para os tripulantes são estabelecidos através da Instrução de Aviação Civil (IAC), n³134-135-0796, sob o título Serviço de Assistência Aeromédica ${ }^{(3)}$.

Observa-se que falta uma padronização, normatização e estabelecimento de protocolos de assistência de enfermagem para o paciente aerorremovido. São essenciais cursos específicos, treinamento permanente e avaliações médicas adequadas em intervalos indicados. Estudos norte-americanos demonstram que tem sido realizados treinamentos com equipes aeromédicas, através de simuladores que funcionam a partir de baterias e compressores de ar dentro de helicópteros, capazes de reproduzir uma situação real de emergência comandados por monitores e laptops $s^{(7)}$.

Dentre os quatro estudos indexados na base de dados Lilacs, observou-se que 50,0\% ( $n=2)$ se referia à área de educação e pesquisa, abordando o perfil, a formação e os aspectos legais do enfermeiro de bordo (1999) e a fisiologia do vôo (2000). No ano de 2003, 100,0\% $(\mathrm{n}=1)$ se referiu à área de cuidados do neonato na remoção aérea. Em 2007 destaca-se $100,0 \%(n=1)$, se referindo à história da enfermagem militar brasileira e resgate aéreo na segunda guerra mundial.
Das 33 publicações indexadas na base Medline, 71,42\% $(n=5)$, no ano de 2001, envolvem a tendência temática cuidado de enfermagem no transporte de crianças, risco neonatal e materno, entubação nasotraqueal cega, classificação de intervenções de enfermagem e paciente crítico. No ano de 2005, $13,33 \%(n=2)$, tratam de educação permanente e pesquisa. Já em 2006, as tendências temáticas educação e pesquisa, cuidado e saúde do trabalhador apresentaram um artigo cada.

Quatro estudos abordam a história da enfermagem aeromédica, salvamentos aéreos, atendimento pré-hospitalar na remoção aérea e enfermagem militar brasileira e resgate aéreo na segunda guerra mundial, nos anos de 1997, 2000, 2003 e 2007. Ressalta-se que a última publicação encontra-se indexada nas duas bases de dados pesquisadas.

Os anos de 1998, 1999, 2006 e 2008, apresentaram uma publicação cada na tendência temática saúde do trabalhador e a de gerenciamento aparece em 2002, totalizando $66,67 \%$ $(n=2)$ e no ano de 2004 , totalizando $33,33 \%(n=1)$.

Na Segunda Guerra Mundial, de 1939 a 1945, destaca-se a presença de Flight Nurses, enfermeiros especializados nesse tipo de atendimento, e que eram membros das Forças Armadas. Essa importância foi confirmada em 1950, durante a Guerra da Coréia, em que sua presença foi obrigatória, atuando na remoção de aproximadamente 10.000 feridos com helicópteros da Marinha ${ }^{(8)}$.

Avaliando o histórico da Primeira e Segunda Guerra Mundial, da Guerra da Coréia e do Vietnã, observa-se muitos feridos, evidenciando a necessidade de um transporte rápido e seguro, com assistência de enfermagem especializada, o que favoreceu o avanço e desenvolvimento técnico-científico das remoções aeromédicas ${ }^{(8)}$. Nesse tipo de remoção, além da tripulação e do médico, a Emergency Nurses Association (ENA), nos EUA, estabelece a necessidade da presença de um enfermeiro treinado, com experiência na assistência a pacientes graves, e que tenha especialização nessa área ${ }^{(3)}$.

No Brasil, a remoção aeromédica teve início na Força Aérea Brasileira (FAB), em que foi criado o Serviço de Busca e Salvamento (SAR), em 1950. Há também as Missões de Misericórdia (MMI), que são meios de Busca e Salvamento, que realizam a remoção de feridos de locais que se caracterizam com escassez de recursos médicos ou outros meios de remoção. Há mais de 40 anos, a FAB possui um sistema de resgate, em regiões de menor infra-estrutura, como a Amazônia e o Centro-Oeste ${ }^{(8)}$.

Já em 1988, no Corpo de Bombeiros Militar do Rio de Janeiro, iniciou-se o transporte aeromédico com o Grupo de Socorro de Emergência (GSE) ${ }^{(3)}$. Em São Paulo, em 1989, foi criado o Projeto Resgate, uma solução conjunta entre Secretaria de Saúde e de Segurança do Estado de São Paulo, no qual a aeronave utilizada foi um helicóptero tipo esquilo ${ }^{(3)}$.

A partir da década de 90, surgem vários serviços de remoção aeromédica privados, para atender à necessidade dos pacientes, porém poucas aeronaves são homologadas pela Agência Nacional de Aviação Civil (ANAC) ${ }^{(3)}$. Para que haja essa homologação, é necessária uma complexa estrutura organizacional, com aeronaves especialmente projetadas para esse fim, com equipamentos especiais e com pessoal capacitado para prestar assistência aos pacientes aerorremovidos. 
No que diz respeito ao ano de publicação e os periódicos em que a produção científica está divulgada no período de 1999 a 2007, destaca-se que os da base Lilacs, encontram-se $75,0 \%(n=3)$ na revista Acta Paulista de Enfermagem e 25,0\% $(\mathrm{n}=1)$ na Revista Brasileira de Enfermagem.

Dos periódicos indexados na Medline, verifica-se que $36,37 \%(n=12)$ dos artigos foram publicados no Air Medical Journal; British Journal of Nursing, Intensive and Critical Care Nursing, Military Medicine, Revista Brasileira de Enfermagem com $6,06 \%(n=2)$ cada e American Journal of Emergency Medicine, Archives Disease Childhood. Fetal and Neonatal Edition, Australian Critical Care, Australian and New Zealand Journal Surgery, Canadian Nurse, Case Maneger, Critical Care Nurse Clinical Noth America, Ed Management, Journal of the Society Pediatric Nurses, Journal of Trauma, Pediatric Emergency Care, Journal of Professional Nursing e Revista da Escola de Enfermagem da USP com 3,03\% $(n=1)$ cada.

Quanto ao delineamento metodológico, observa-se na Tabela 3 que $51,51 \%(n=17)$ das publicações da base Medline são estudos qualitativo etnográfico, seguido por fenomenológico $27,27 \%(n=9)$, quantitativo não experimental $15,15 \%$ $(n=5)$ e quase-experimental 6,07\% $(n=2)$. Na Lilacs, 50,0\% $(n=2)$, qualitativo etnográfico e qualitativo fenomenológico e quantitativo não experimental com 25,0\% $(n=1)$ cada.

Vale ressaltar que a realização dos estudos com delineamento metodológico qualitativo tem sido amplamente utilizada na enfermagem em diversas áreas do conhecimento ${ }^{(9)}$. Autores identificaram que $50,0 \%$ das pesquisas publicadas no período de 1977 a 2001 apresentaram delineamento qualitativo, sendo a fenomenologia o mais utilizado pelos pesquisadores ${ }^{(10)}$.

$\mathrm{Na}$ pesquisa experimental o pesquisador é um agente ativo, mais do que um observador passivo. Os experimentos não ocorrem necessariamente em laboratórios, podendo ser conduzidos em qualquer ambiente. A manipulação, o controle e a randomização são características do delineamento de pesquisa experimental. Na pesquisa quase-experimental há o envolvimento da manipulação, porém não possui características de randomização ou de grupo controle, ou ambas. Na pesquisa não-experimental a (não) manipulação da variável independente deve ser considerada eticamente e é considerada descritiva, cuja finalidade é observar, descrever e documentar os aspectos da situação, tendendo a ser realista e de difícil crítica ${ }^{(6)}$.

Os estudos descritivos são a categoria mais ampla de desenhos não experimentais em Enfermagem. São utilizados para buscar informações precisas, construindo um quadro de um fenômeno com as características da situação em foco; enfatizam mais a amplitude do que a profundidade das informações. No estudo ora realizado observou-se que esse tipo de pesquisa foi necessário para diagnosticar várias situações de trabalho ${ }^{(5)}$.

Em relação ao delineamento metodológico faz-se necessário compreender a associação de sua escolha com o objeto a ser estudado e não à preferência do pesquisador ${ }^{(11)}$. Ou seja, dependendo do objeto estudado, são identificadas variações sobre o delineamento metodológico usado(12).

No cenário das pesquisas qualitativas, a opção por um método e suas técnicas de coleta, incluindo o tratamento dos dados, é importante que o pesquisador obtenha uma visão global dos resultados, dando ênfase ao corpus que compreende as observações e as entrevistas ${ }^{(12)}$.

\section{CONCLUSÕES}

Este estudo mostra que a produção científica acerca do transporte aéreo de pacientes, em bases de dados indexadas, iniciou-se em 1997, intensificando-se em 2001, com prevalência de publicação internacional. As tendências temáticas que mais apareceram foram o cuidado de enfermagem na remoção aeromédica e educação e pesquisa na enfermagem aeroespacial.

Atualmente, a remoção aérea vem crescendo consideravelmente, devido à rapidez, pois se percorrem grandes distâncias em intervalos de tempo menores, e pelas condições de trânsito, principalmente nas grandes cidades. Destaca-se a escassa regulamentação no que se refere às atribuições do enfermeiro de bordo, enfatizando a complexidade e a especificidade na remoção dos pacientes do local do acidente até o ambiente hospitalar.

É evidente que esse tipo de remoção, ainda recente em nosso país, requer treinamento específico e constante atualização, visando uma assistência de enfermagem de qualidade.

Neste estudo confirmou-se que o delineamento metodológico qualitativo tem sido amplamente empregado nas publicações da enfermagem. Ressalta-se a importância da inclusão do conteúdo acerca de enfermagem aeroespacial nos cursos de graduação e pós-graduação em enfermagem, já que esta é uma área de atuação que vem crescendo consideravelmente.

Considera-se que existem ainda muitas lacunas na produção de conhecimento sobre o tema. Acredita-se que pesquisas que analisem esta interface, muito contribuem para a qualificação do cuidado de enfermagem aeroespacial.

Tabela 2 - Distribuição dos resumos na base de dados Lilacs e Medline segundo o delineamento metodológico.

\begin{tabular}{|c|c|c|c|}
\hline & Medline & Lilacs & Total \\
\hline Tipos de pesquisa & $\mathbf{n}$ & $\mathbf{n}$ & $\mathbf{n}$ \\
\hline Quantitativa Experimental & - & - & - \\
\hline Quantitativa Quase-experimental & 2 & - & 2 \\
\hline Quantitativa Não experimental & 5 & 1 & 6 \\
\hline Qualitativa / Fenomenologia & 9 & 1 & 10 \\
\hline Qualitativa / Teoria Fundamentada nos Dados & - & - & - \\
\hline Qualitativa / Etnografia & 17 & 2 & 19 \\
\hline Total & 33 & 4 & 37 \\
\hline
\end{tabular}




\section{REFERÊNCIAS}

1. Judge T. Breathing easier - good news from air medicine. Critical Care 2008; 12(4):164.

2. Mannarino L, Timerman S. Transporte terrestre e aéreo do paciente crítico. Rev Soc Cardiol Estado de São Paulo 1998; 8(4):866-78.

3. Thomaz RR, Miranda MFB, Souza GAG, Gentil RC. Enfermeiro de bordo: uma profissão no ar. Acta paul. enferm. 1999;12(1):86-96.

4. Cervo AL, Bervian PA. Metodologia científica. 4a.ed. São Paulo: MAKRON Books; 1996.

5. Lo Biondo-Wood G, Haber J. Pesquisa em enfermagem: métodos, avaliação crítica e utilização. 4a.ed. Rio de Janeiro: Guanabara-Koogan; 1998.

6. Polit DF, Beck CT, Hungler BP. Fundamentos de pesquisa em enfermagem métodos avaliação e utilização. 5a.ed. Porto Alegre (RS): Artmed; 2004.

7. Wright SW, Lindsell CJ, Hinckley WR, Williams A, Holland $\mathrm{C}$, Lewis $\mathrm{CH}$, et al. Hight fidelity medical simulation in the difficult environment of a helicopter: feasibility, self-efficacy and cost. BMC Medical Education [Internet]. 2006 Oct [cited 2006 Oct 5]; 6:[49 screens]. Available from: http://www.biomedcentral.com/1472-6920/6/49

8. Bernardes MMR, Teixeira GL. Enfermeiras do Exército Brasileiro no transporte aéreo de feridos: um desafio enfrentado na 2a. Guerra Mundial. Rev Bras Enferm 2007; 60(1): 68-72.

9. Demo P. Pesquisa qualitativa: busca de equilíbrio entre forma e conteúdo. Rev. Latino-Am. Enfermagem 1998; 6 (2): 89-104.

10. Oliveira EB, Lisboa MTL. Análise da produção científica da vertente saúde do trabalhador de enfermagem: subjetividade e trabalho. Rev. enferm. UERJ 2004; 12 (1): 24-9.

11. Almeida VCF, Damasceno MMC, Araújo TL. Saúde do trabalhador de saúde: análise sobre o tema. Rev Bras Enferm 2005; 58 (3): 335-40.

12. 12 - Campos CJG, Turato ER. Análise de conteúdo em pesquisas que utilizam metodologia clínico-qualitativa: aplicação e perspectivas. Rev. Latino-Am. Enfermagem 2009; 17 (2): 259-264. 